\title{
Nanoemulsions of Essential Oil Constituents
}

\section{Joyce Nirmala $\mathbf{M}^{*}$, Arjun Rakesh and Nagarajan $\mathbf{R}$}

Department of Chemical Engineering, Indian Institute of Technology Madras, Chennai 600036 , India

\section{Antimicrobial Nanoemulsions}

Essential oils (EOs) are hydrophobic liquids which are derived from aromatic plants. These concentrated liquids are known for their varied biological applications and are therefore widely commercialised across the world. Various methods such as solvent extraction, distillation, expression and florasol extraction are employed to extract essential oils. Apart from its popularity in the fragrance and flavouring industry, EOs are known for their applications in pharmaceuticals. Phenolic constituents, amongst many other functional compounds, lend these natural extracts antimicrobial properties $[1,2]$ by having a destructive impact on the cell membrane of target microorganisms. These constituents include, but are not limited to, carvacrol, eugenol, camphor, cinnamaldehyde, linalool, geraniols, thymol, citral, and terpineol. The efficacies of EOs as medicines are, however, constrained by their poor aqueous solubility, high reactivity and volatility, which are attributable to the lipophilic nature of these essential oils [3]. Nanoemulsification technique is used to overcome these drawbacks, as it leverages the ability of nanoemulsions to deliver the oils which are otherwise insoluble in water. This, along with enhanced surface area for interaction (because of the smaller droplet size), greater thermodynamic stability, improved bioavailability and ease of formation, makes nano emulsification an ideal augmentation to the pharmaceutical applications of EOs [4].

Essential oils, being a combination of 60 or more compounds [5], are not an exclusive collection of beneficial chemical compounds and comprise of undesirable components. Also, studies on oregano essential oil suggest that its antibacterial properties are largely dominated by constituent compounds carvacrol and thymol, with both contributing additively to that of oregano essential oil (OEO) in their inhibition of Pseudomonas aeruginosa and Staphylococcus aureus. Separate formulations of carvacrol and thymol stand to provide the same efficacy of using OEO, while at the same time addressing flavour constraints that the Essential Oil is known for [6]. This suggests that a further breakdown of EOs into their useful constituents and consequent emulsification of the same will enhance their scope for application. There are published reports on the antimicrobial efficacy of nanoemulsified constituents of essential oils. Two food-grade nanoemulsions, loaded with geraniol and linalool, were studied to exhibit promising results against $E$. coli and L. innocua in a meat simulation system [7]. Optimized formulations of nanoemulsions of carvacrol made using spontaneous emulsion were observed to inhibit yeast strains, Zygosaccharomyces bailii (ZB), Saccgromyces cerevisae (SC), Brettanomyces bruxellensis (BB) and Brettanomyces naardenesis (BN) [8]. Eugenol nanomulsions prepared using food-grade emulsifiers gum arabic and lecithin showed similar results to pure eugenol in inhibiting Listeria monocytogenes and Salmonella enteritidis, while exhibiting better stability [9]. Eugenol nanoemulsions have also been observed to exhibit antibacterial activity against $S$. aureus [10] and antifungal activity against Fusarium oxysporum $f$. sp. vasinfectum and phytotoxicity on cotton seeds [11]. Citral is another constituent whose use, although efficient against microorganisms, is constrained because of its instability and hydrophobicity in normal condition. Ultrasonication method was used to nanoemulsify this component and the resultant nanoemulsion were observed to exhibit strong antibacterial properties against $L$. monocytogenes and $S$. aureus [12]. These studies suggest that individual constituents of essential oils are equally effective in their antimicrobial properties and can help overcome specific constraints that are generally faced in using essential oils for pharmaceutical purposes.

\section{References}

1. Mahmoud SS, Croteau RB (2002) Strategies for transgenic manipulation of monoterpene biosynthesis in plants. Trends Plant Sci 7: 366-373.

2. Cosentino S, Tuberoso Cl, Pisano B, Satta ML, Mascia V, et al. (1999) In-vitro antimicrobial activity and chemical composition of Sardinian thymus essential oils. Lett Appl Microbiol 29: 130-135.

3. Burt S (2004) Essential oils: their antibacterial properties and potential applications in foods-a review. Int J Food Microbiol 94: 223-253.

4. Tenjarla S (1999) Microemulsions: an overview and pharmaceutical applications. Crit Rev Ther Drug Carrier Syst 16: 461-521.

5. Senatore $F(1996)$ Influence of harvesting time on yield and composition of the essential oil of a thyme (Thymus pulegioides L.) growing wild in Campania (Southern Italy). J Agric Food Chem 44: 1327-1332.

6. Lambert RJ, Skandamis PN, Coote PJ, Nychas GJ (2001) A study of the minimum inhibitory concentration and mode of action of oregano essential oil thymol and carvacrol. J Appl Microbiol 9: 453-462.

7. Balta I, Brinzan L, Stratakos AC, Linton M, Kelly C, et al. (2017) Geraniol and linalool loaded nanoemulsions and their antimicrobial activity. Bulletin UASVM Animal Science and Biotechnologies 74: 2.

8. Chang Y, McLandsborough L, McClements DJ (2013) physicochemical properties and antimicrobial efficacy of carvacrol nanoemulsions formed by spontaneous emulsification. J Agric Food Chem 61: 8906-8913.

9. Hu Q, Gerhard H, Upadhyaya I, Venkitanarayanan K, Luo Y (2016) Antimicrobial eugenol nanoemulsion prepared by gum arabic and lecithin and evaluation of drying technologies Int. J Biol Macromol 87: 130-140.

10. Ghosh V, Mukherjee A, Chandrasekaran N (2004) Eugenol-loaded antimicrobia nanoemulsion preserves fruit juice against, microbial spoilage. Colloids Surf $B$ Biointerfaces 114: 392-397.

11. Abd-Elsalam KA, Khokhlov AR (2015) Eugenol oil nanoemulsion: antifungal activity against Fusariumoxysporum f. sp. vasinfectum and phytotoxicity on cotton seeds. Appl Nanosci 5: 255-265.

12. Lu WC, Huang DW, Wang CC, Yeh CH, Tsai JC, et al. (2017) Preparation characterization, and antimicrobial activity of nanoemulsions incorporating citral essential oil. J Food Drug Anal 26: 82-89.

*Corresponding author: Joyce Nirmala $M$, Department of Chemical Engineering, Indian Institute of Technology Madras, Chennai 600 036, India, Tel: 9677174200; E-mail: joycegitz@gmail.com

Received: March 25, 2018; Accepted: April 02, 2018; Published: April 09, 2018

Citation: Nirmala MJ, Rakesh A, Nagarajan R (2018) Nanoemulsions of Essentia Oil Constituents. J Nanomed Nanotechnol 9: 495. doi: 10.4172/2157-7439.1000495

Copyright: (c) 2018 Nirmala MJ, et al. This is an open-access article distributed under the terms of the Creative Commons Attribution License, which permits unrestricted use, distribution, and reproduction in any medium, provided the original author and source are credited. 